BRiEF SUMmaAY of Prescribing Information-Betore preseribing, please consult complette
Prescribing Intarmation.

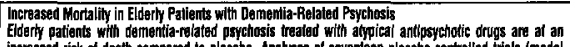

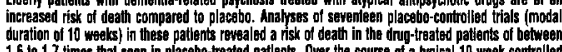

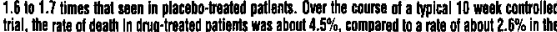

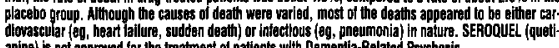

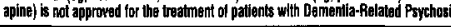

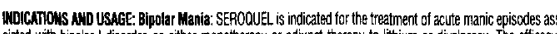

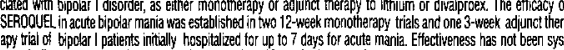

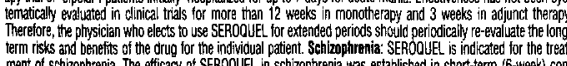

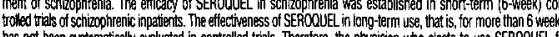

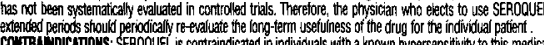

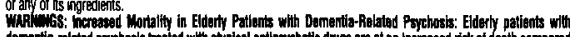

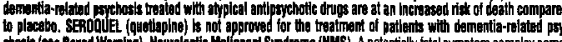

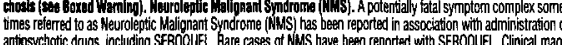

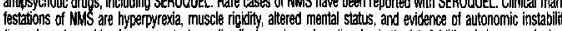

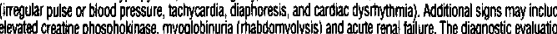

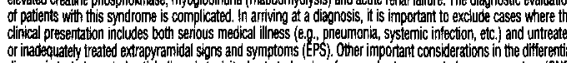

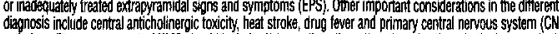

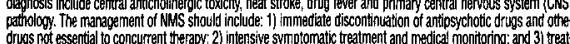

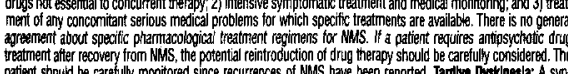

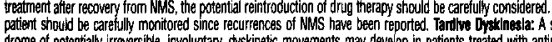

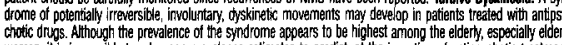

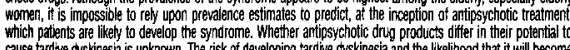

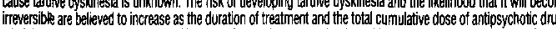

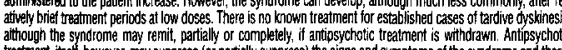

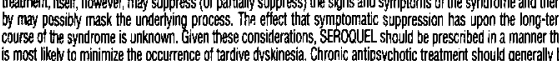

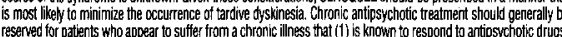

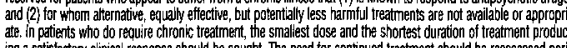

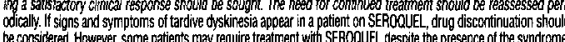

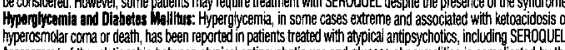

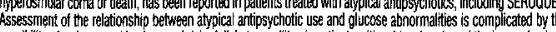

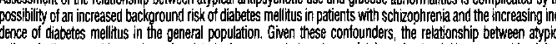

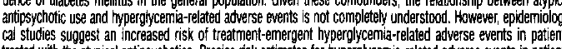

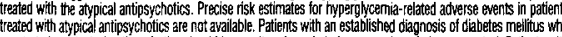

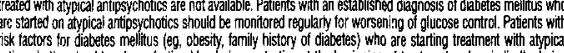

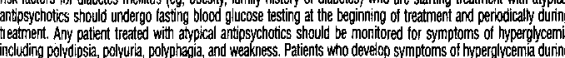

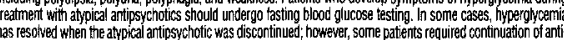

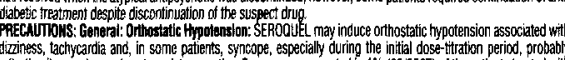

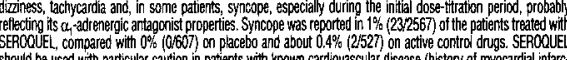

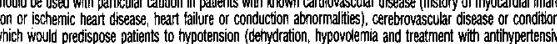

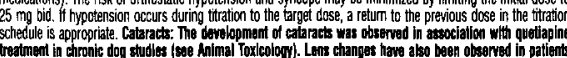

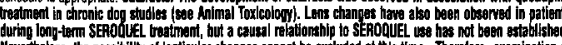

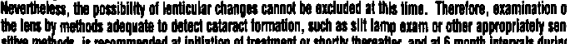

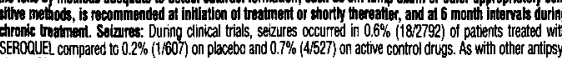

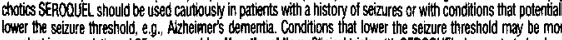

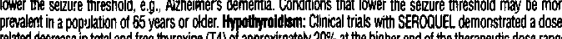

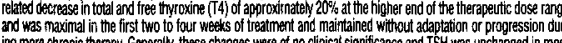

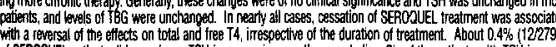

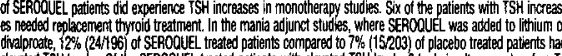

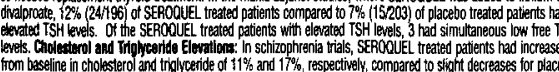

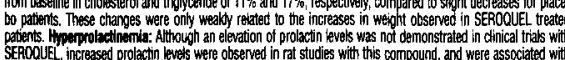

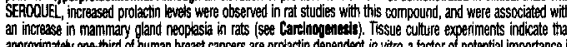

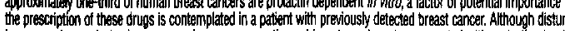

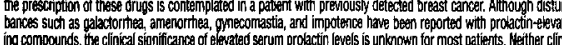

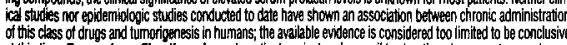

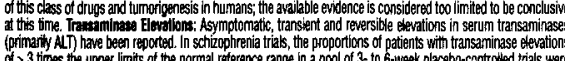

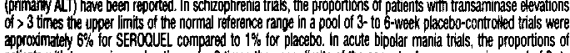

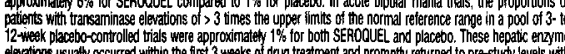

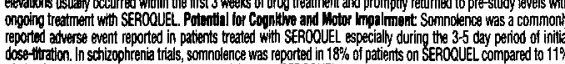

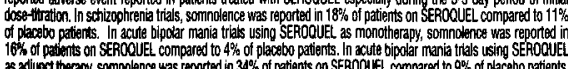

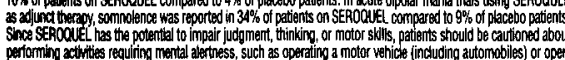

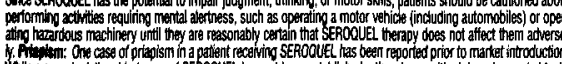

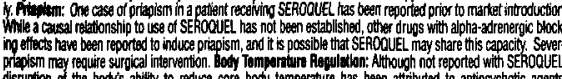

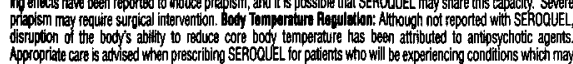

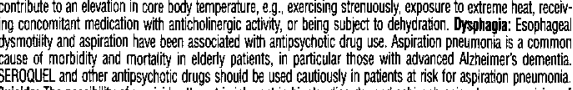

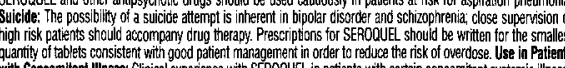

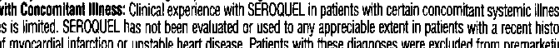

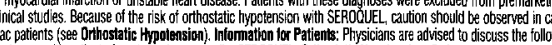

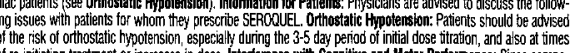

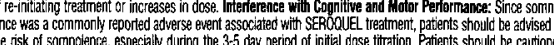

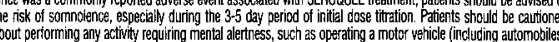

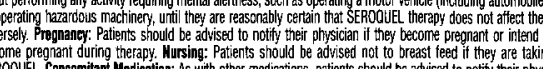

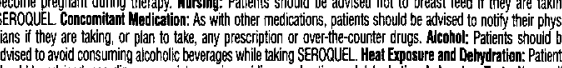

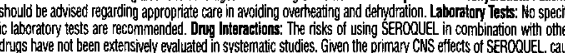

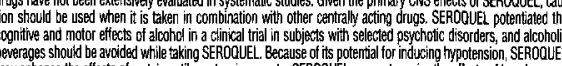

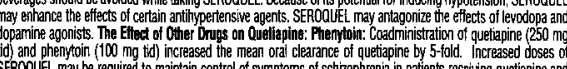

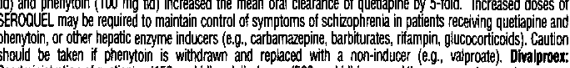

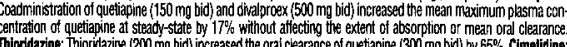

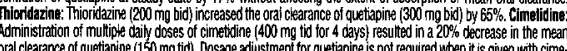

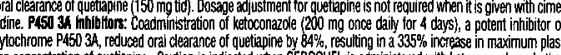

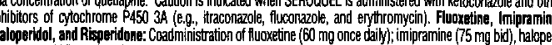

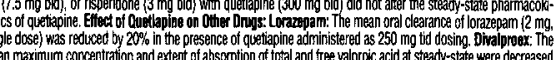

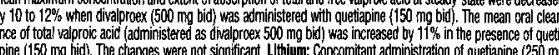

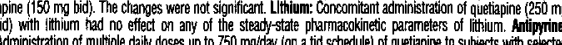

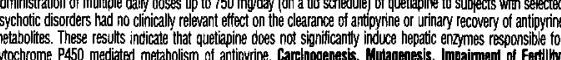

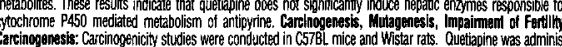

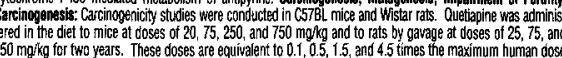

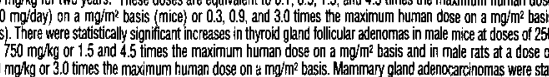

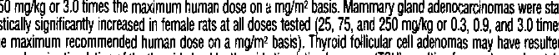

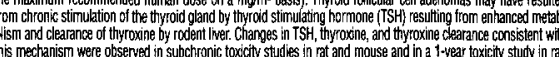

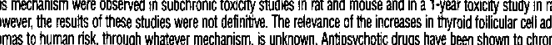

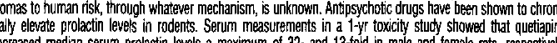

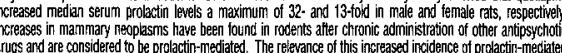

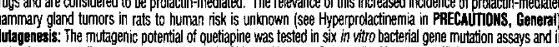

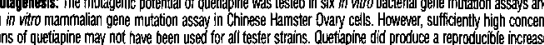

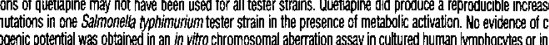

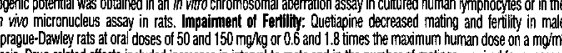

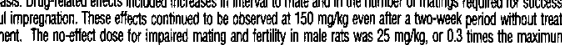

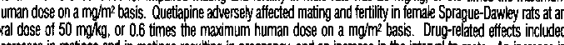

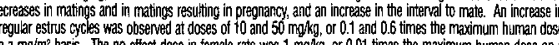

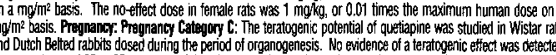

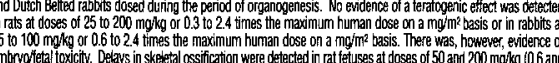

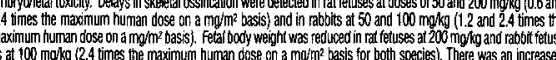

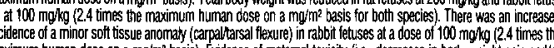

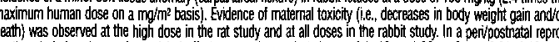

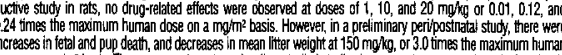

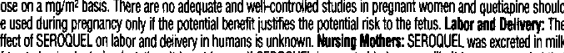

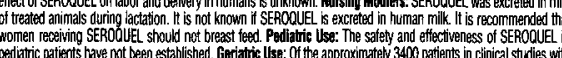

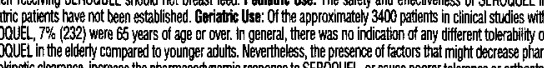

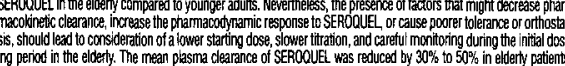

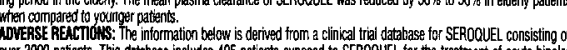

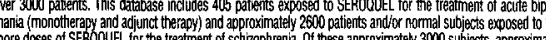

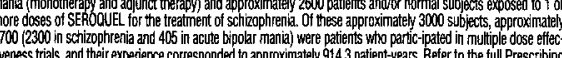

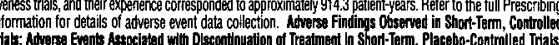

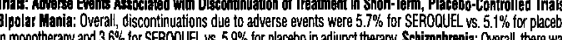

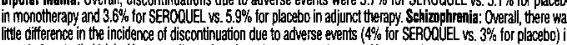

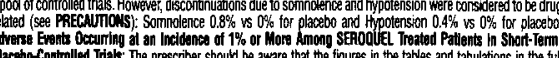

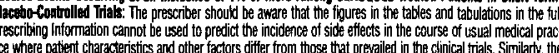

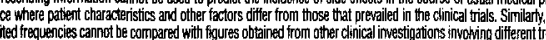

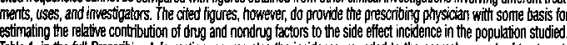

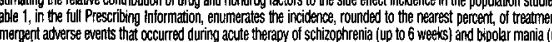

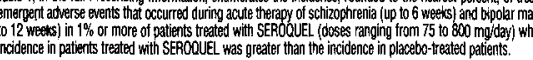

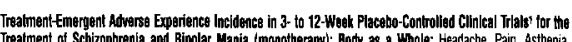

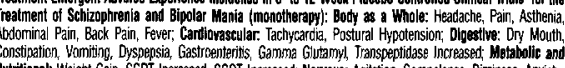

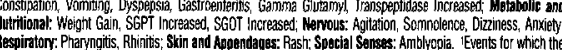

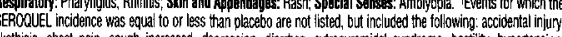

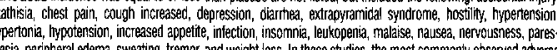

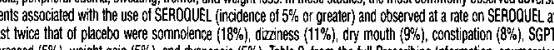

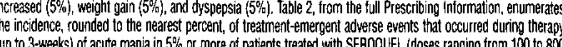

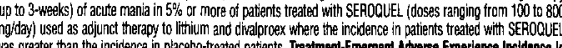

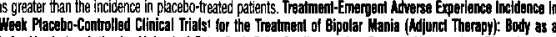

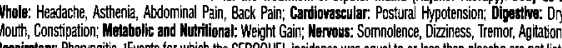

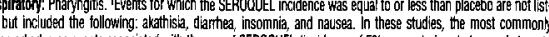

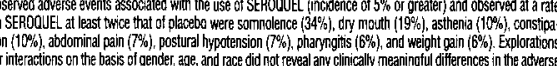

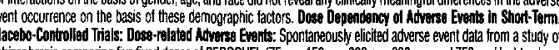

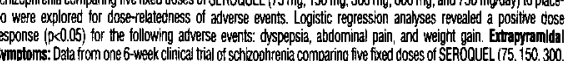

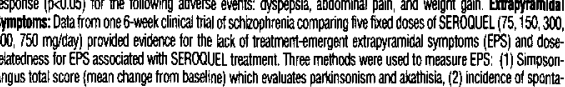

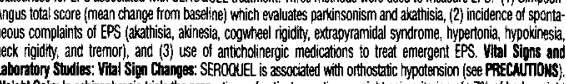

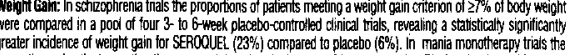

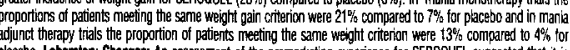

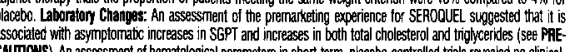

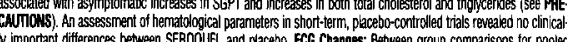

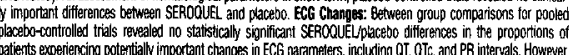

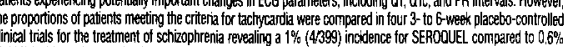

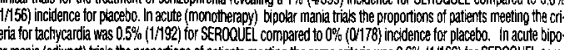

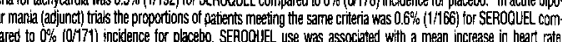

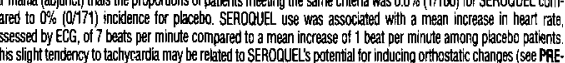

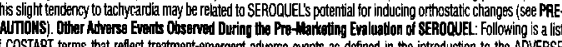

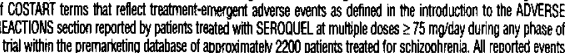

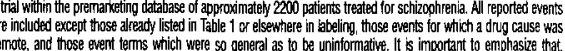

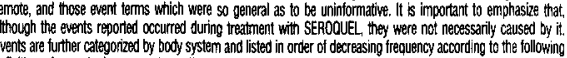

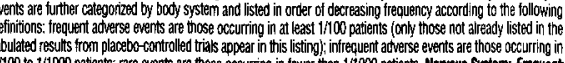

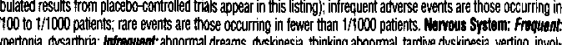

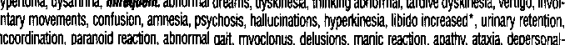

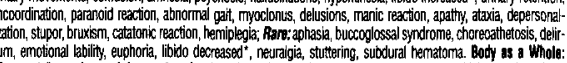

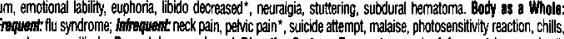

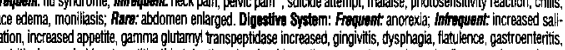

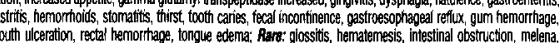

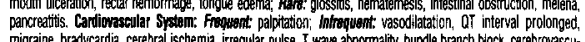

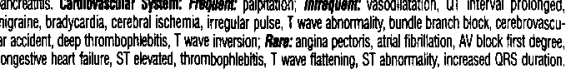

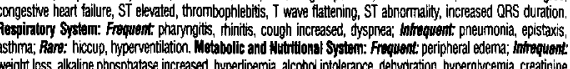

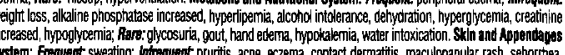

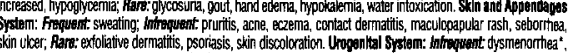

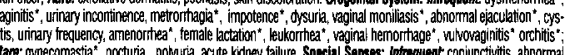

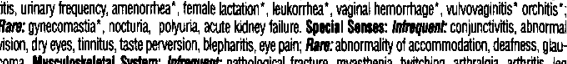

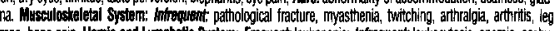

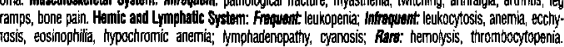

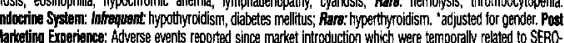

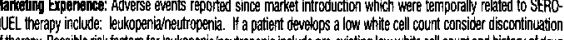

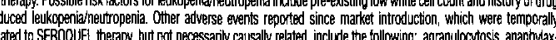

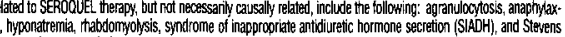

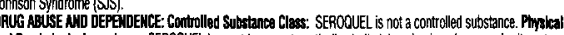

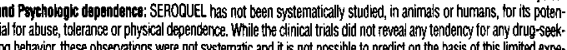

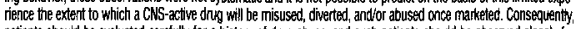

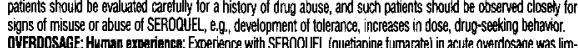

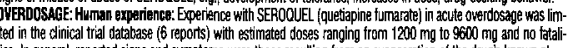

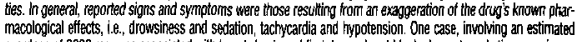

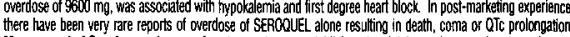

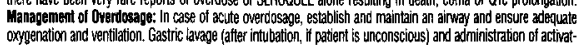

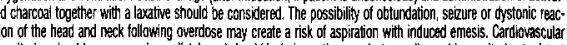

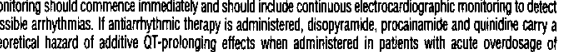

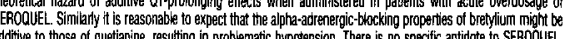

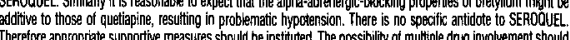

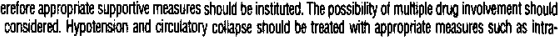

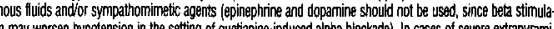

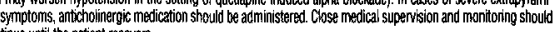

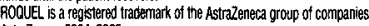

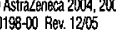

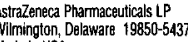




\section{reasons to prescribe}

\section{New 50-mg and 400-mg tablets}

- $50 \mathrm{mg}$ for simpler dosing during initiation

- $400 \mathrm{mg}$ for an easier way to achieve higher doses*

- SEROOUEL is indicated for the treatment of acute manic episodes associated with bipolar I disorder, as either monotherapy or adjunct therapy with lithium or divalproex, and the treatment of schizophrenia. Patients should be periodically reassessed to determine the need for continued treatment

- Elderly patients with dementia-related psychosis treated with atypical antipsychotic drugs are at an increased risk (1.6 to 1.7 times) of death compared to placebo $(4.5 \%$ vs $2.6 \%$, respectively). SEROQUEL is not approved for the treatment of patients with dementia-related psychosis

- Prescribing should be consistent with the need to minimize the risk of tardive dyskinesia. A rare condition referred to as neuroleptic malignant syndrome has been reported with this class of medications, including SEROQUEL

- Hyperglycemia, in some cases extreme and associated with ketoacidosis, hyperosmolar coma, or death, has been reported in patients treated with atyplcal antipsychotics, including SEROQUEL. Patients starting treatment with atypical antipsychotics who have or are at risk for diabetes should undergo fasting blood glucose testing at the beginning of and during treatment. Patients who develop symptoms of hyperglycemia should also undergo fasting blood glucose testing

- Precautions include the risk of seizures, orthostatic hypotension, and cataract development

- The most commonly observed adverse events associated with the use of SEROQUEL in clinical trials were somnolence, dry mouth, dizziness, constipation, asthenia, abdominal pain, postural hypotension, pharyngitis, SGPT increase, dyspepsia, and weight gain

"The safety of doses above $800 \mathrm{mg} /$ day has not been evaluated in clinical trials. In the elderly and in patients with hepatic impairment, consideration should be given to a lower starting dose, a slower rate of dose titration, careful monitoring during the initial dosing period, and a lower target dose.

All atypical prescriptions: Total prescriptions. Jan. 05-Dec. 05. New prescriptions. Sept. 04-Dec. 05. IMS Health. National Prescription Audit.

Please see Brief Summary of Prescribing Information on adjacent page.

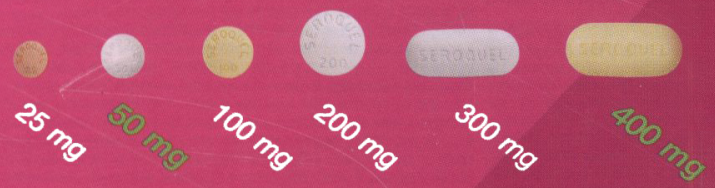

Tablets shown are not actual size

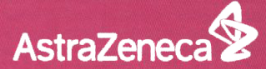

Astrazeneca Pharmaceuticals LP

(c) 2006 AstraZeneca Pharmaceuticals LP. All rights reserved.

SEROQUEL is a registered trademark of the AstraZeneca group of companies.

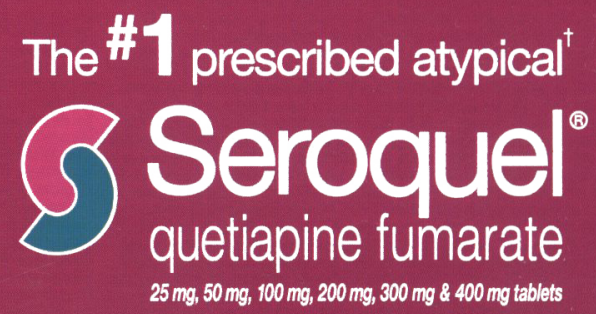

236660

$1 / 06$

WWW.SEROQUEL.COM 